\title{
Omega-3 Fatty Acids Protect Fatty and Lean Mouse Livers After Major Hepatectomy
}

\author{
Michael Linecker, MD, ${ }^{*}$ Perparim Limani, MD, ${ }^{*}$ Patryk Kambakamba, MD, ${ }^{*}$ Philipp Kron, MD, \\ Christoph Tschuor, MD, ${ }^{*}$ Nicolas Calo, MSc, $\dagger$ Michelangelo Foti, PhD, $\dagger$ Jean-François Dufour, MD, $\ddagger$ \\ Rolf Graf, PhD, ${ }^{*}$ Bostjan Humar, PhD, ${ }^{*}$ and Pierre-Alain Clavien, MD, PhD*
}

Objective: The aim of this study was to assess the effect of $\Omega 3$ fatty acids $(\Omega 3 \mathrm{FA})$ on fatty and lean liver in hepatic surgery.

Background: The global spread of energy-dense diets has led to an endemic rise in fatty liver disease and obesity. Besides metabolic pathologies, steatosis enhances hepatic sensitivity to ischemia reperfusion (I/R) and impedes liver regeneration (LR). Steatosis limits the application of liver surgery, still the main curative option for liver cancer. $\Omega 3 \mathrm{FA}$ are known to reverse steatosis, but how these lipids affect key factors defining surgical outcomes- that is, I/R, LR, and liver malignancy-is less clear.

Methods: We established a standardized mouse model of high fat diet (HFD)induced steatosis followed by $\Omega 3 F A$ treatment and the subsequent assessment of $\Omega 3 F A$ effects on $\mathrm{I} / \mathrm{R}, \mathrm{LR}$, and liver malignancy ( $\mathrm{n}=5 /$ group), the latter through a syngeneic metastasis approach. Fatty liver outcomes were compared with lean liver to assess steatosis-independent effects. Nonparametric statistics were applied.

Results: $\Omega 3 F A$ reversed HFD-induced steatosis and markedly protected against I/R, improved LR, and prolonged survival of tumor-laden mice. Remarkably, these beneficial effects were also observed in lean liver, albeit at a smaller scale. Notably, mice with metastases in fatty versus lean livers were associated with improved survival.

Conclusions: $\Omega 3$ FA revealed multiple beneficial effects in fatty and lean livers in mice. The improvements in I/R injury, regenerative capacity, and oncological outcomes await confirmatory studies in humans.

Keywords: $\Omega-3$ polyunsaturated fatty acids, ischemia-reperfusion injury, liver regeneration, liver surgery

(Ann Surg 2016;xx:xxx-xxx)

From the *Swiss HPB and Transplantation Center, University Hospital Zurich, Zurich, Switzerland; †Department of Cellular Physiology and Metabolism, Faculty of Medicine, University of Geneva, Geneva, Switzerland; and $\ddagger$ Hepatology Unit, Department of Clinical Research, University Hospital Bern, Bern, Switzerland.

Reprints: Pierre-Alain Clavien, MD, PhD, Department of Surgery, University Hospital Zurich, Raemistrasse 100, CH-8091 Zurich, Switzerland. E-mail: clavien@access.uzh.ch.

ML, BH, MF, JFD did the experimental design. ML, PL, PKa, PKr, CT, NC did the data acquisition and analyses. BH, ML, MF, JFD, RG did the data interpretation. BH, ML did the study concept and design. BH, ML, PAC did the manuscript writing and critical revision. BH, PAC provided funding for this study. All authors approved the final version.

Bostjan Humar and Pierre-Alain Clavien shared senior authorship.

The project was supported by grants from the Sinergia grant from the Swiss National Science Foundation 310030B_150042 and CRSII3_141798/1, from the Clinical Research Priority Program University of Zurich (CRPP Liver Tumors) and from the Liver and Gastrointestinal Disease Foundation (LGID).

All authors contributing to this manuscript declare that they have nothing to disclose. They do not have a conflict of interest with respect to this manuscript.

Supplemental digital content is available for this article. Direct URL citations appear in the printed text and are provided in the HTML and PDF versions of this article on the journal's Web site (www.annalsofsurgery.com).

Copyright (c) 2016 Wolters Kluwer Health, Inc. All rights reserved.

ISSN: 0003-4932/14/26105-0821

DOI: $10.1097 /$ SLA.0000000000001968
T he increasing consumption of fat-laden diets is putting a strain on the liver. Many societies experience an endemic rise in obesity and the metabolic syndrome (MS), often already apparent in children and young adults. ${ }^{1}$ Nonalcoholic fatty liver disease (NAFLD) is the hepatic manifestation of the MS and is thought to underlie its hallmark insulin resistance and its associated cardiovascular risks. ${ }^{2}$

Surgical removal remains the only curative option for liver tumors with an increased risk of liver failure in the presence of hepatic steatosis. ${ }^{3}$ To prevent blood loss during liver resection, portal inflow is temporarily occluded, leading to an ischemic period that may cause significant liver injury upon reperfusion. Steatosis not only enhances the liver's sensitivity toward ischemic insults but also impedes its regenerative capacity in terms of both repairing ischemic injury and compensating for lost volume following resection., ${ }^{4,5}$ Together, these features lead to an elevated rate of postoperative complications that restrict surgical options in patients with fatty liver. ${ }^{5}$

Simple and well-tolerated means to negate the impact of liver fat on surgical outcomes are highly desired. Omega-3 polyunsaturated fatty acids $(\Omega 3 \mathrm{FA})$, most known for their potential benefits to cardiovascular health, ${ }^{6}$ may combine many properties sought for liver surgery. Although mechanisms underlying $\Omega 3 \mathrm{FA}$ action are incompletely understood, observations such as the coupling of enhanced energy turnover with anti-inflammatory mechanisms are likely related to the potent anti-steatotic effects described for $\Omega 3 \mathrm{FA}$ in liver. ${ }^{7-9}$ Of note, $\Omega 3$ FA have been reported to suppress steatosis also in a mouse model of diet-induced obesity ${ }^{10}$ and in NAFLD patients. ${ }^{11}$ The anti-inflammatory properties of $\Omega 3 F A$ further translate into an efficient protection of animal liver from ischemiareperfusion (I/R) injury. We have shown that $\Omega 3 F A$ reduce $I / R$ injury in lean mouse liver via engagement of Gpr120, which imprints an anti-inflammatory M2 polarization phenotype onto Kupffer cells. ${ }^{12}$ Likewise, $\Omega 3$ FA were also effective against I/R in the $o b /$ $o b$ model of NAFLD. ${ }^{13}$ In addition, $\Omega 3$ FA seem to improve recovery of lean liver from resection-induced liver failure, or of fatty liver after standard resection. ${ }^{14,15}$ Whether such effects are indirect (eg, by protecting from injury) or direct (eg, by promoting hepatocyte proliferation) remains unclear, but the resulting benefits can also be observed in the clinic. ${ }^{16}$ Finally, $\Omega 3$ FA have been associated with anti-cancerous properties in a variety of neoplasms. ${ }^{17}$ The effects of $\Omega 3 F$ A on colorectal liver metastasis (CRLM) - the most common indication for liver surgery in the Western world-are unclear. In a syngeneic rat model, $\Omega 3$ FA promoted CRLM development, while in another study-based on the same rats and cancer cells- $\Omega 3 \mathrm{FA}$ revealed beneficial effects. ${ }^{18,19}$

The effects on steatosis, hepatic $\mathrm{I} / \mathrm{R}$, liver recovery after resection, and liver tumors together suggest a fallow potential of $\Omega 3 F A$ to improve outcomes in liver surgery. What remains unknown is (i) whether $\Omega 3 F A$ 's beneficial effects are simply due to fat reduction, (ii) whether $\Omega 3 F A$ can directly promote parenchymal regeneration after resection, and (iii) whether $\Omega 3 F A$ do counteract the formation or growth of liver tumors, particularly in a fatty background. Moreover, it is not established whether the single effects 
are bound to a given model or rather are a general feature of $\Omega 3 \mathrm{FA}$. To address these questions, we designed a standardized mouse model of fatty liver surgery based on a high fat diet (HFD). This model enabled us to study the effects of dietary $\Omega 3 F A$ on steatosis, I/R, liver regeneration, and tumor risk in the settings of pre-existing NAFLD compared with a lean background. Our findings strongly encourage the pre-operative use of $\Omega 3 F A$ to expand the application of surgery for fatty liver.

\section{METHODS}

\section{Animals and Diets}

All animal experiments were approved by the Veterinary Office of Zurich, Switzerland. Male mice (C57BL6, Harlan) were purchased at the age of 8 to 10 weeks and kept on a 12-hour day/night cycle. After accommodation for 1 week, animals were either exposed to HFD (total energy $22 \mathrm{MJ} / \mathrm{kg}, 60 \mathrm{~kJ} \%$ from fat and $8.4 \mathrm{~kJ} \%$ sucrose) or a control $\operatorname{diet}(\mathrm{CD}$, total energy $15 \mathrm{MJ} / \mathrm{kg}, 11 \mathrm{~kJ} \%$ from fat without sucrose) for 6 weeks (ssniff, Soest, Germany). During treatment, overall calories in HFD were reduced by $3 \mathrm{MJ} / \mathrm{kg}$ to mimic mild pre-operative calorie restriction, and for $\Omega 3 F A$ treatment, fish oil (mainly eicosapentaenoic acid, EPA, and docosahexaenoic acid, DHA) replaced $45 \%$ of total fat while maintaining $8.4 \mathrm{~kJ} \%$ sucrose (Supplementary Figure 1A, http:// links.lww.com/SLA/B84). Each treatment group included 5 animals, with a total of 180 animals used for the experimental setup.

\section{Lipid Quantification Using Small Animal MRI and Chemical Analysis}

To assess liver fat content longitudinally in vivo, a smallanimal 4.7-Tesla magnetic resonance imaging (MRI) scanner (Bruker BioSpin MRI, Inc., Massachusetts) was used (Supplementary Figure 1B, http://links.lww.com/SLA/B84). ${ }^{20}$ Liver fat was chemically quantified by the Vanillin method. ${ }^{21}$

\section{Surgical Procedures}

Isoflurane inhalation (2\% to $4 \%$ ) anesthesia (Pittman-Moore, Chicago, IL) including subcutaneous injection of buprenorphine $(0.1 \mathrm{mg} / \mathrm{kg})$ was used for all surgical interventions. For ischemia/ reperfusion (I/R) experiments, $68 \%$ hepatic ischemia was induced as described. ${ }^{22}$ Animals were sacrificed 6 hours after reperfusion, a time point that well reflects injury levels at later reperfusion times. ${ }^{22}$ Hepatectomies (68\% for normal liver regeneration, $86 \%$ for inducing liver failure) were performed as reported. ${ }^{23}$ Omegaven (parenteral fish oil emulsion; Fresenius Kabi AG, Oberdorf, Switzerland), was intravenously (i.v.) injected 1 hour before hepatectomy at $0.2 \mathrm{~g} / \mathrm{kg}$. Cell solutions $\left(10^{5} / 100 \mu l\right.$ phosphate-buffered saline [PBS] $)$ of exponentially growing MC-38 colorectal cancer cells were injected into the portal vein under selective clamping as described, ${ }^{24}$ inducing tumors limited to the right and caudate lobes.

\section{Cell Culture}

MC-38 cells were cultured as reported. ${ }^{24}$ Cultures were tested negative for mycoplasma at their onset (PCR Mycoplasma Test Kit; PromoCell Gmbh, Heidelberg, Germany).

\section{Histological Analyses}

Immunohistochemistry on archived liver sections was performed for Ki67 (Abcam ab16667) and pH3 (Abcam ab92628) as described. ${ }^{23}$ Quantification was done by blindly counting nuclear positivity in 10 random visual fields.

\section{Serum and Tissue Analysis}

Serum alanine aminotransferase (ALT) and aspartate aminotransferase (AST) levels were measured using a multiple biochemical analyzer (Dri-Chem 4000i; Fujifilm, Tokyo, Japan). Serum Hmgb1 levels were assessed by ELISA (Shino-Test, Japan, via IBL International). The malondialdehyde assay (MDA) from Oxis (Bioxytech \#21012) was used to estimate lipid peroxidation.

\section{Quantitative Real-Time PCR (qPCR)}

Quantitative real-time polymerase chain reactions were run on a ABI Prism 7000 Sequence Detector System (PE Applied Biosystems, Rotkreuz, Switzerland) using following TaqMan gene expression assays (PE Applied Biosystems, Rotkreuz, Switzerland): Illb (Mm00434228_m1), Emr1 (Mm00802530_m1), Tnfa (Mm00443258_m1), and normalization control $18 S r R N A .^{23}$

\section{Statistical Analysis}

Each group consisted of $n=5$ unless otherwise stated. Data are presented as mean $\pm \mathrm{SD}$. Group differences were assessed by Mann-Whitney testing throughout the manuscript. Survival was assessed by Kaplan-Meier analysis. Significance levels are categorized by stars $\left({ }^{*} P<0.05,{ }^{* *} P<0.01,{ }^{* * *} P<0.001\right)$. Statistical analyses were performed using GraphPad Prism 6.0 (GraphPad Software, Inc., La Jolla, CA).

\section{RESULTS}

\section{Equicaloric $\Omega 3 F A$ Substitution for the Reduction of High Fat Diet Induced Steatosis}

The experimental set up is shown in Figure 1A. Six weeks of HFD feeding led to a gain in mean bodyweight from $21.5 \mathrm{~g}( \pm 1.3)$ to $34.3 \mathrm{~g}( \pm 2.9)$, rising to $40.6 \mathrm{~g}( \pm 3.9)$ after 10 weeks in the HFD control group (Figure 1B). In the HFD- $\Omega 3 F A$ group, bodyweight increased to $39.2 \mathrm{~g}( \pm 4.9)$ indicating that $\Omega 3 \mathrm{FA}$ treatment has little effect on diet-induced obesity $(P=0.686)$. Liver weight grew from $0.9 \mathrm{~g}( \pm 0.1)$ to $1.8 \mathrm{~g}( \pm 0.2)$ after 6 weeks and $1.9 \mathrm{~g}( \pm 0.3)$ after 10 weeks in the HFD group, but was decreased by $\Omega 3 F A$ from $1.8 \mathrm{~g}$ $( \pm 0.2 \mathrm{~g})$ to $1.5 \mathrm{~g}( \pm 0.1, P=0.016)$. Both groups had a comparable food intake during treatment (HFD $360.4 \mathrm{~g} /$ cage vs HFD- $\Omega 3 F A$ $356.5 \mathrm{~g} / \mathrm{cage}$ ). On histology, lipid accumulation was seen in hepatocytes after 6 weeks of HFD. After 10 weeks, severe microsteatosis with rare signs of macrosteatosis (lipid droplets, displaced hepatocyte nuclei) was present in the HFD group, while steatosis was markedly diminished in the HFD- $\Omega 3 F A$ group and marginal in the CD group (Figure 1C). Histology revealed no signs of fibrosis or significant immune infiltration. No significant elevations in hepatic lipid peroxidation, serum Hmgb1 levels, and inflammatory markers were noted in the HFD group (Supplementary Figure 2, http://links. lww.com/SLA/B84). Therefore, 10 weeks of HFD induce simple steatosis in mouse liver.

Chemical analysis confirmed steatosis, with lipid contents after 6 weeks being $41.1( \pm 12.2)$ and $14.2 \mu \mathrm{g} / \mathrm{mg}( \pm 2.4, P=0.002)$ in liver tissue from HFD and CD animals, respectively (Figure 1D). Similarly, liver fat assessed by MRI was significantly elevated after 6 weeks HFD compared with CD, further increased after 10 weeks of HFD, but was drastically reduced in the HFD- $\Omega 3 F A$ group (Figure 1D). Therefore, MRI was used to longitudinally monitor steatotic development and treatment effects in vivo.

Altogether, our HFD mouse feeding protocol designed to mimic western diet induces obesity and simple steatosis, while $\mathrm{CD}$ feeding is associated with little gain in body or liver weight [6 weeks: body $26.9 \mathrm{~g}( \pm 2.0) /$ liver $1.3 \mathrm{~g}( \pm 0.2)$; 10 weeks: 28.6 $( \pm 2.6) / 1.5 \mathrm{~g}( \pm 0.3)$; CD- $\Omega 3$ FA 10 weeks: $30.9( \pm 3.4) / 1.2 \mathrm{~g}( \pm 0.1)$; $P=0.530$ for bodyweight, $P=0.111$ for liverweight]. Four weeks of $\Omega 3 F A$ treatment via dietary fat substitution are associated with a reduction in steatosis to levels similar to those in CD-fed animals 


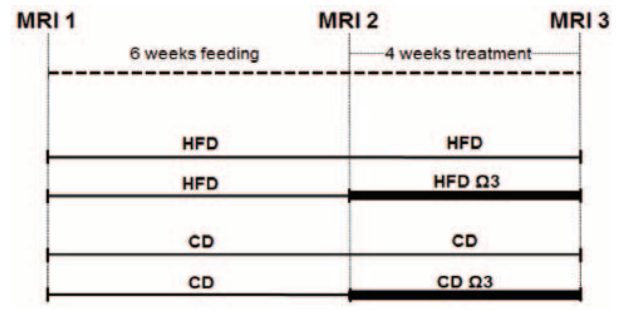

A

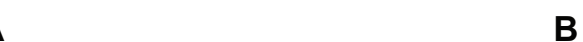

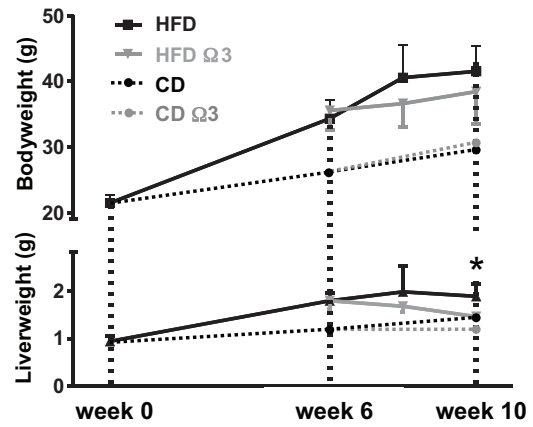
week 10

FIGURE 1. Antisteatotic effects of $\Omega 3 F A$ in a model of HFD-induced fatty liver disease. A, Experimental design showing schedules of HFD/CD feeding and $\Omega 3 F A$ treatment. Effects were assessed subsequent to the treatment unless otherwise stated. B, Body and liver weight during the feeding and treatment periods. C, Liver histology of mice exposed to CD or HFD for 6 weeks, and to another 4 weeks of HFD or HFD along with $\Omega 3 F$ A substitution. Pale cytoplasmic spots represent lipid vesicles. D, Chemical analysis of hepatic fat content after differential feeding, and MRI assessment of liver fat content after the feeding/ treatment period. For all figures: $\mathrm{n}=5 /$ group unless otherwise stated; ${ }^{*} P<0.05$, ${ }^{* *} P<0.01,{ }^{* * *} P<0.001$.
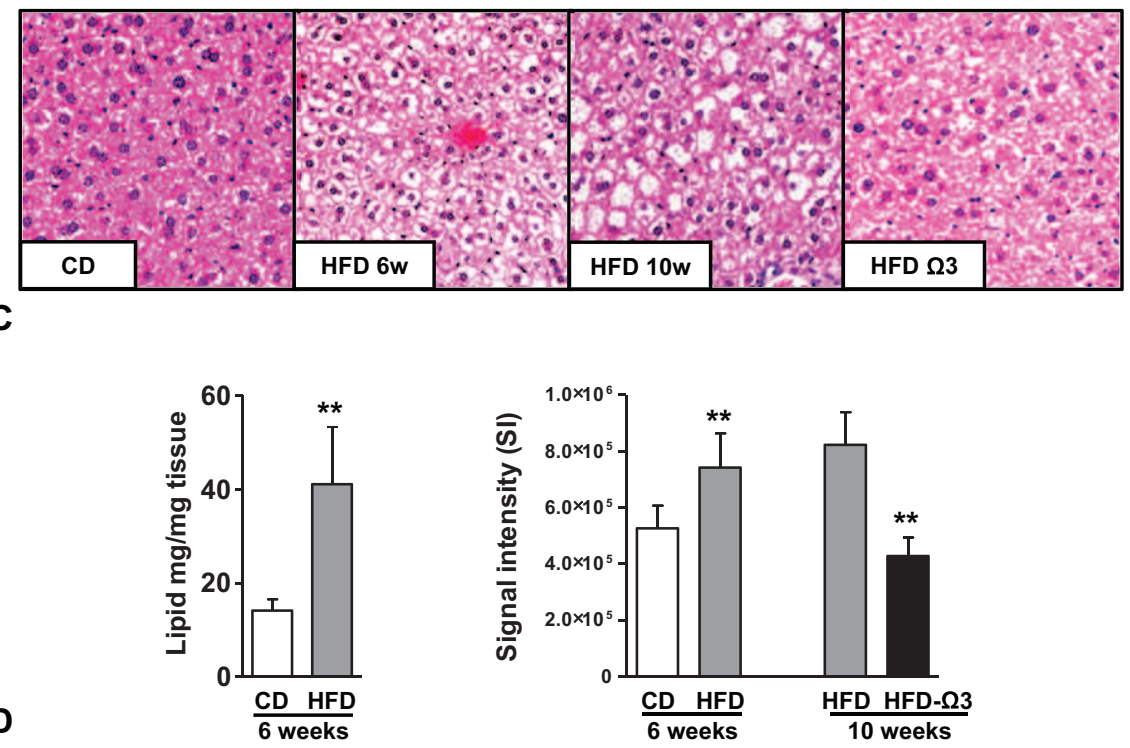

C

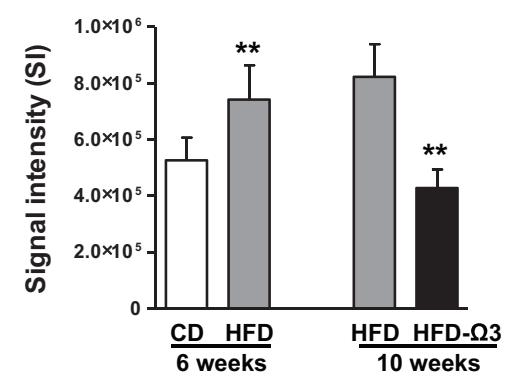

[SI $427813( \pm 80,847)$ vs SI $526322( \pm 65,886), P=0.056)$, Figure 1D], consistent with the reported $\Omega 3$ FA-defattening effects. $^{7-11}$ Therefore, our HFD protocol yields a clinically relevant model to study how pre-operative $\Omega 3 F A$ treatment impacts surgical outcomes (I/R injury, liver regeneration, and malignant risk) complicated through fatty liver.

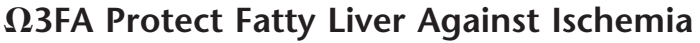 Reperfusion}

Steatosis increases the sensitivity of liver toward I/R. ${ }^{13} \Omega 3 F A$ can protect against $\mathrm{I} / \mathrm{R}$ in genetic models of NAFLD; however, whether protection can be also achieved in diet-induced steatosis is unknown.

Following treatment, HFD and HFD- $\Omega 3 F A$ mice were exposed to partial hepatic ischemia for 60 minutes. Six hours after reperfusion, ${ }^{22}$ liver injury was assessed via multiple parameters. Necrosis on histology was visibly reduced in the HFD- $\Omega 3 F A$ versus the HFD group (Figure 2A), as was sinusoidal plugging indicated by stuck erythrocytes. Likewise, marked reductions through $\Omega 3 \mathrm{FA}$ treatment were noted for the injury markers ALT/AST, and for Hmgb1, released by necrotic cells and propagating parenchymal injury following reperfusion (Figure $2 \mathrm{~B}$ ) ${ }^{25}$ Therefore, $\Omega 3 \mathrm{FA}$ treatment of mouse liver with diet-induced steatosis offers effective protection against hepatic I/R.

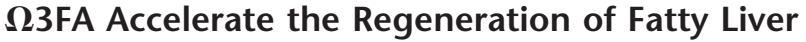 After Resection}

Lipid accumulation in hepatocytes diminishes hepatoregenerative capacities, thereby increasing the likelihood of postoperative complications including liver failure. ${ }^{4,5}$ After $68 \%$ hepatectomy $(68 \% \mathrm{HX})$ in lean liver, the original liver weight is gradually regained (by $50 \%$ after 48 hours, $70 \%$ after 96 hours) and completely restored after 1 week. ${ }^{23}$ HFD-induced steatosis was associated with a delayed liver weight regain after 68\% HX $(45 \%$ after 48 hours, 50\% after 96 hours). $\Omega 3 \mathrm{FA}$ treatment improved regeneration patterns to those seen in lean mice ( $50 \%$ after 48 hours, $70 \%$ after 96 hours), suggesting that $\Omega 3$ FA can normalize regenerative capacity (Figure $3 \mathrm{~A}$ ). $\Omega 3 \mathrm{FA}$ treatment also improved the liver-to-body weight ratio (LW/BW) after resection (Figure 3A), however less pronounced than for percentage increase, because $\Omega 3 F A$ differentially affect liver versus body weight (Figure 1B).

Counts for Ki67, marking all cycling cells, were elevated in the HFD- $\Omega 3 F A$ versus HFD group at most times examined after $68 \% \mathrm{HX}$ (Figure 3B). To assess cell cycle progression, we examined $\mathrm{pH} 3$, a differential marker for cells in the $\mathrm{G}_{2}$ (weak nuclear positivity) and $\mathrm{M}$ (bold nuclear positivity) cell cycle phases. ${ }^{23}$ The ratio of bold versus total $\mathrm{pH} 3$ counts thus reflects the proportion of mitotic cells among cells past the $S$-phase. Compared with the HFD group, $\Omega 3 F A$ treated liver displayed a significantly increased $\mathrm{pH} 3$ ratio at 72 and 96 hours postresection (Figure 3C). Therefore, $\Omega 3$ FA treatment of 

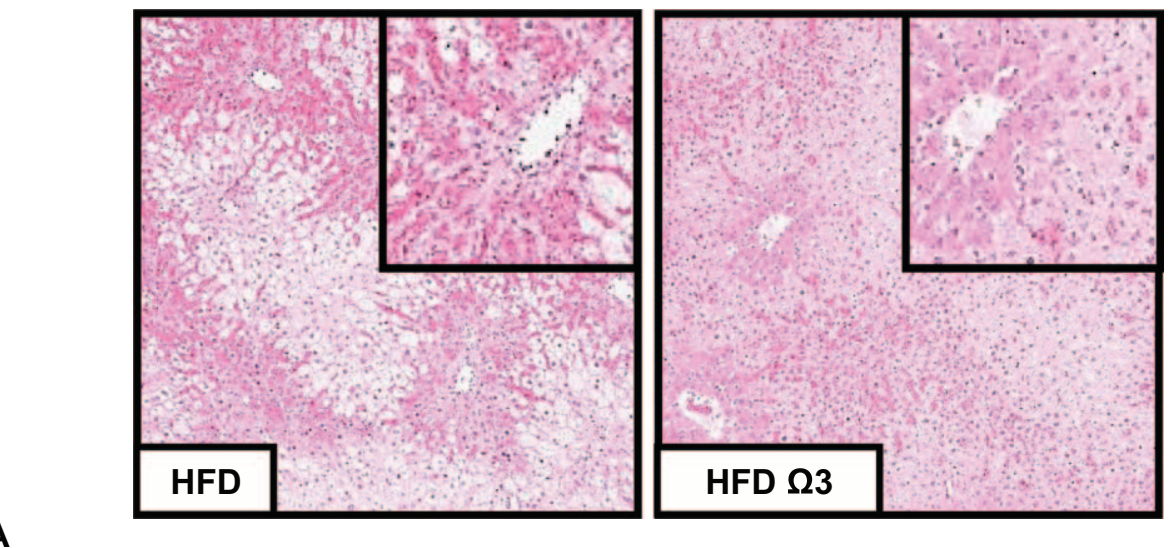

A

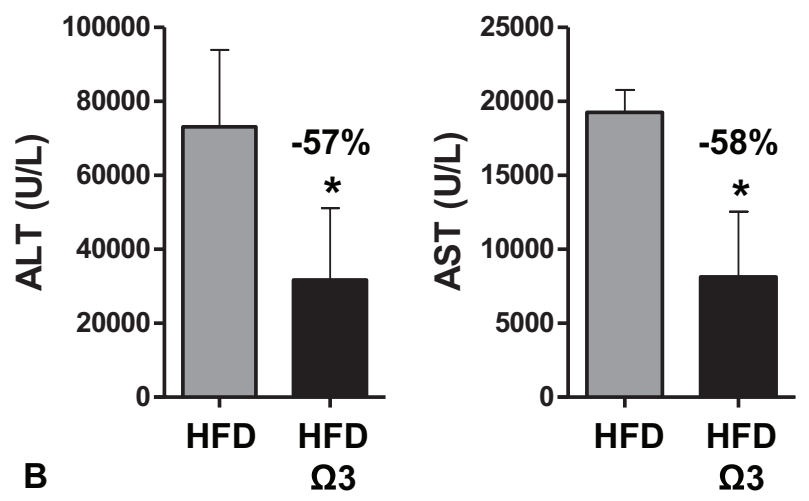

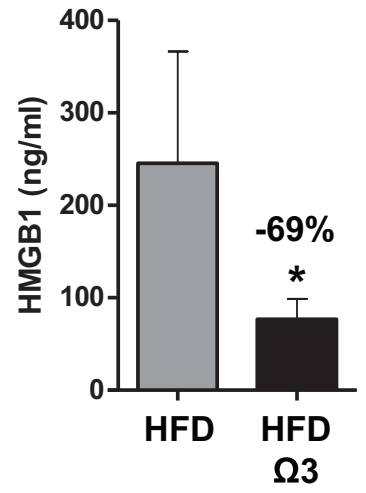

FIGURE 2. $\Omega 3$ FA effects on I/R injury in fatty liver 6 hours after reperfusion. A, Histological necrosis. B, Serum ALT, AST, and Hmgb1 levels. fatty liver promotes entry of hepatocytes into cell cycle and their mitotic progression at times when hepatocellular division usually is completed. $^{23}$

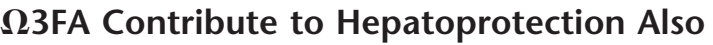 Independent of Fat Reduction}

To investigate whether the protection of fatty liver against I/R through $\Omega 3 \mathrm{FA}$ is solely due to steatosis reduction, ${ }^{26}$ experiments were repeated in mice fed on CD for 6 weeks, and then exposed or not to $\mathrm{CD}$ diet enriched in $\Omega 3 \mathrm{FA}$.

After 10 weeks feeding, CD mice present with little steatosis in their liver (Figure 1C, D). Accordingly, a 4-week $\Omega 3 F A$ led to an insignificant reduction in lipid content (Figure 4A). When $\Omega 3 F A-C D$ animals were subjected to hepatic ischemia, injury levels were reduced by about a half compared with the CD group (Figure 4B, C).

We conclude that in addition to the reduced sensitivity toward $\mathrm{I} / \mathrm{R}$ following fat reduction, $\Omega 3 \mathrm{FA}$ also exert steatosis-independent protection against hepatic ischemia.

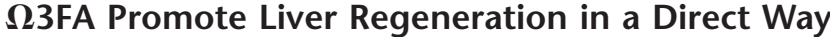

To determine whether $\Omega 3 \mathrm{FA}$ can improve liver regeneration also independent of fat reduction, ${ }^{26} \mathrm{CD}$-fed mice treated or not with $\Omega 3 F A$ were subjected to $68 \% \mathrm{HX}$.

Regenerative improvements through $\Omega 3 \mathrm{FA}$ were less pronounced in lean versus fatty liver. The percentage gain in liver weight was significantly raised by $\Omega 3 \mathrm{FA}$ at 96 hours postresection time, while LW/BW tended to be increased (Figure 5A). Ki67 and
$\mathrm{pH} 3$ counts confirmed the improvements following $\Omega 3 \mathrm{FA}$ treatment, with more hepatocytes in cycle and with accelerated mitotic progression at 96 hours (Figure 5B, C). To provide additional evidence for a pro-regenerative effect of $\Omega 3 \mathrm{FA}$ in lean liver, we tested an $86 \% \mathrm{HX}$ model of regenerative deficiency due to defective cell cycle progression. ${ }^{23} \Omega 3 \mathrm{FA}$ (or vehicle ${ }^{12}$ ) were i.v. injected as an emulsion (Omegaven) 1 hour before surgery to reveal their direct effects. Examining 96 hours post $86 \% \mathrm{HX}$, when regeneration is most delayed, ${ }^{23}$ Omegaven increased liver weight (Figure 5D), consistent with a direct pro-regenerative effect on liver.

\section{及3FA Counteract Colorectal Liver Metastases in a Syngeneic Model}

Given the pro-regenerative effects of $\Omega 3 \mathrm{FA}$, their impact on liver malignancy was unclear.

To estimate potential effects of $\Omega 3 F$ F on hepatic malignancy, we used a syngeneic, orthotopic mouse model of CRLM, wherein MC-38 cancer cells are injected in the portal vein under selective clamping, leading to lobe-restricted tumor growth that is the major determinant of survival. ${ }^{24}$ We tested 2 settings (Figure 6A): the direct impact of $\Omega 3 \mathrm{FA}$ treatment on the establishment and growth of CRLM (cancer cell injection before treatment), and the indirect impact, that is, how CLRM development is affected after liver has been treated with $\Omega 3 F A$ (injection after treatment, similar to surgeryinduced disease recurrence). Tumor inoculation before treatment led to a median survival of 36 days (from injection) in HFD animals (Figure 6B), with median liver weight at $4.3 \mathrm{~g}( \pm 1.4)$. In contrast, 
FIGURE 3. $\Omega 3 F A$ effects on regeneration of fatty liver after $68 \%$ hepatectomy. A, Percent liver weight after hepatectomy in HFD and HFD- $\Omega 3 F A$ mice. B, LW/BW after hepatectomy. C, Percentage of Ki67-positive hepatocytes after hepatectomy. Photographs show representative examples of Ki67 staining. D, Differential $\mathrm{pH} 3$ counts after hepatectomy. The ratio displays the number of hepatocytes with bold $\mathrm{pH} 3$ staining (marking $\mathrm{M}$ cells, see examples marked by arrows) divided by the number of all pH3-positive cells (marking $\mathrm{G}_{2}$ and $\mathrm{M}$ cells).

FIGURE 4. $\Omega 3 F A$ effects on $I / R$ injury in CD liver. A, MRI signal intensity representing liver fat in mice on a CD and treated or not with $\Omega 3 F A$ (see Fig. 1D for HFD liver). Note the small effect of $\Omega 3 F A$ treatment on the MRI signal. B, Histological necrosis and C, Serum ALT, AST, and Hmgb1 levels at 6 hours after reperfusion.
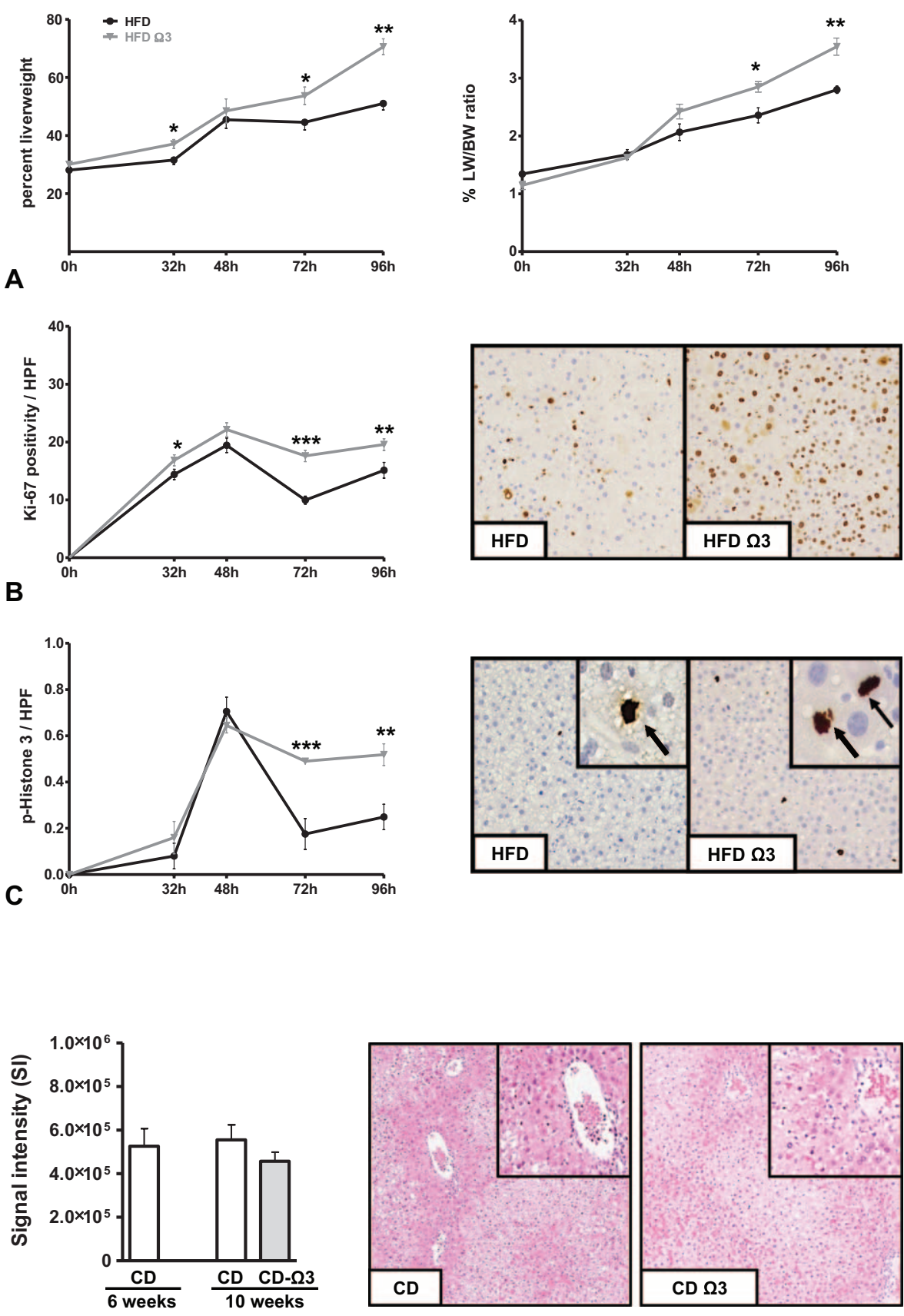

A

B
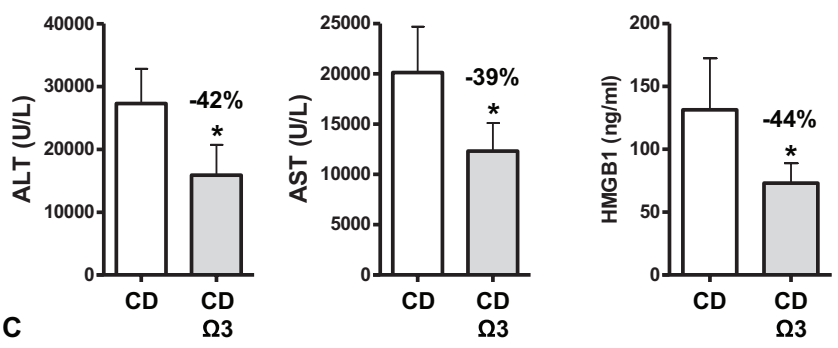
A

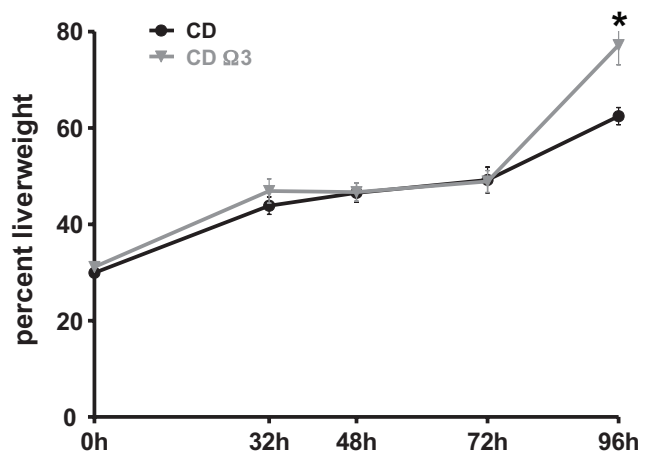

B
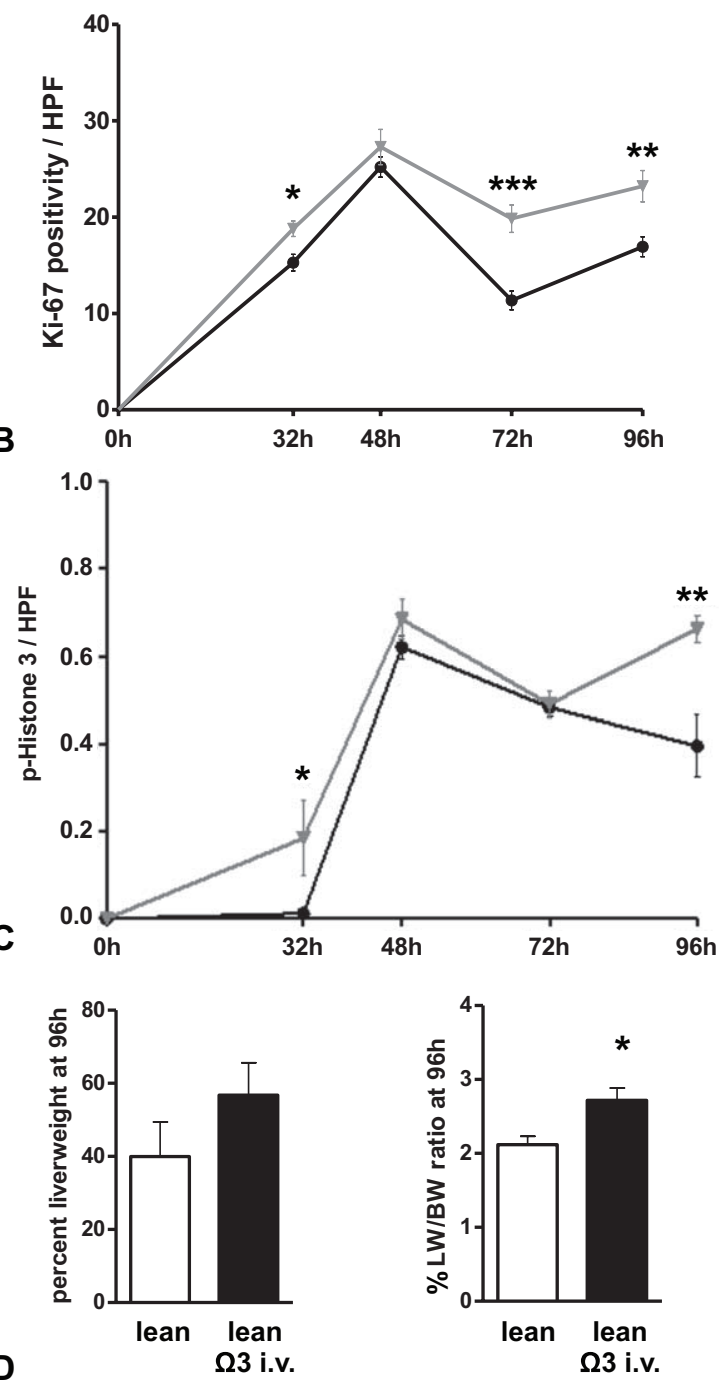
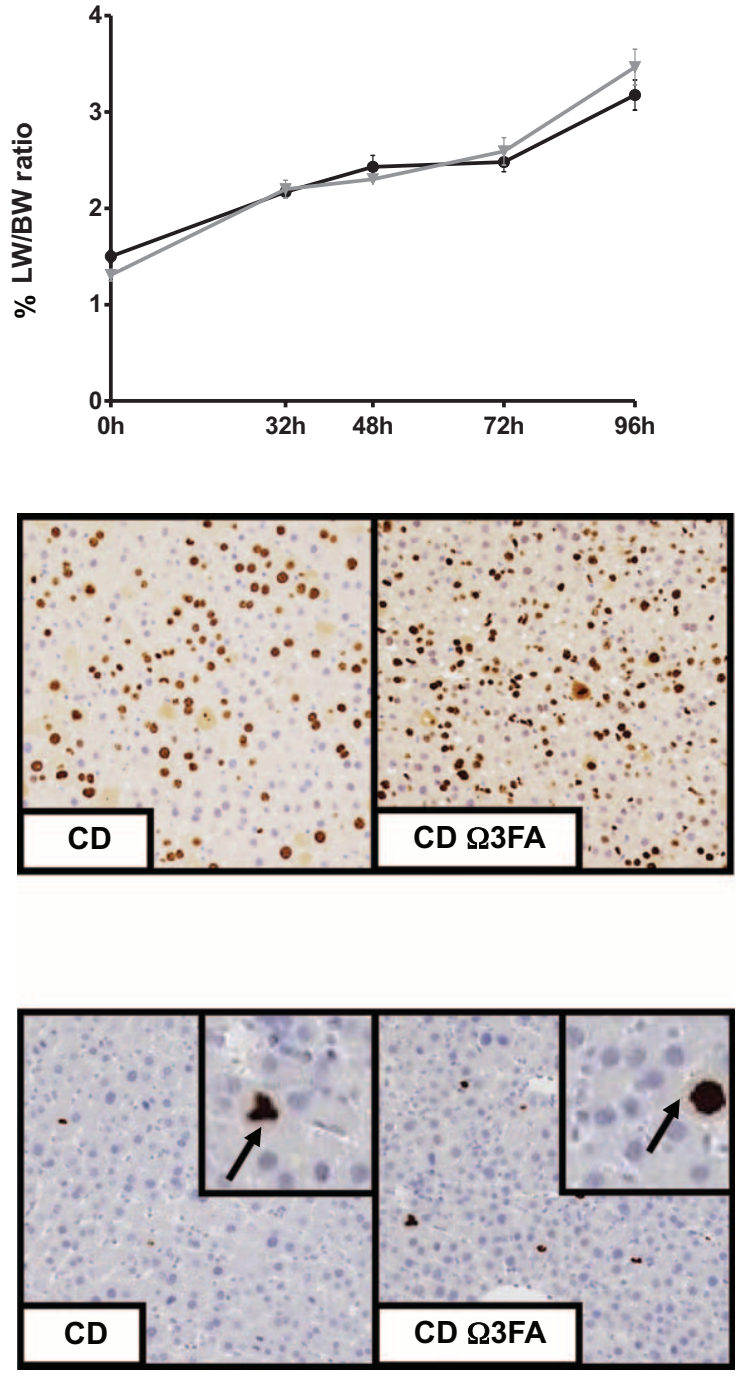

FIGURE 5. $\Omega 3 F A$ effects on regeneration of $C D$ liver after hepatectomy. A, Percent liver weight and LW/BW after hepatectomy in $C D$ or CD- $\Omega 3$ FA mice. B, Percentage of Ki67-positive hepatocytes after hepatectomy. C, Differential pH3 counts after hepatectomy. Arrows mark pH3-positive mitotic cells. D, Percent liver weight and LW/BW at 96 hours after extended (86\%) hepatectomy with/ without concomitant $\Omega 3 F A$ injection.

survival in HFD- $\Omega 3 F A$ animals was 59 days $(P=0.07)$ and liver weight $3.8 \mathrm{~g}( \pm 0.4)$, suggesting $\Omega 3 F A$ inhibit CRLM in pre-existing steatosis. For tumor inoculation after treatment, survival after injection was 40 days [liver weight $5.2 \mathrm{~g}( \pm 1.7)$ in the HFD group and was extended to 47 days $(P=0.156$; liver weight $5.6 \mathrm{~g}( \pm 1.1)]$ in the HFD- $\Omega 3$ FA group (Figure $6 C$ ). To better assess the latter differences, liver weight ( $\mathrm{n}=3$ /group) was measured at day 30 postinjection and was $2.9 \mathrm{~g}( \pm 0.5)$ for HFD and $1.5 \mathrm{~g}( \pm 0.3), P=0.100)$ for 
A
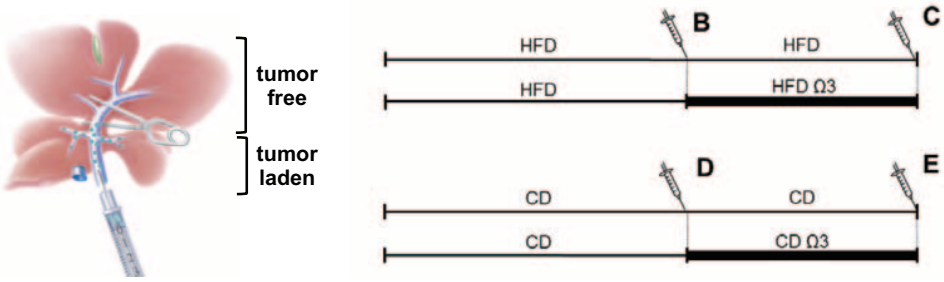

MC-38 cell injection before treatment

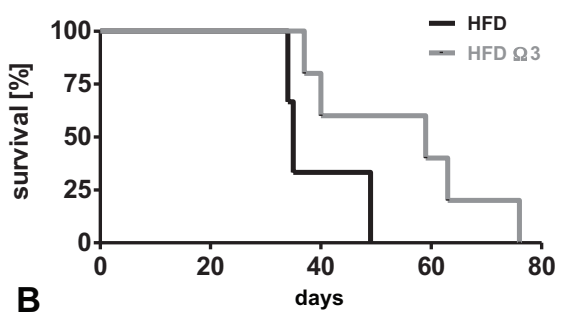

MC-38 cell injection before treatment

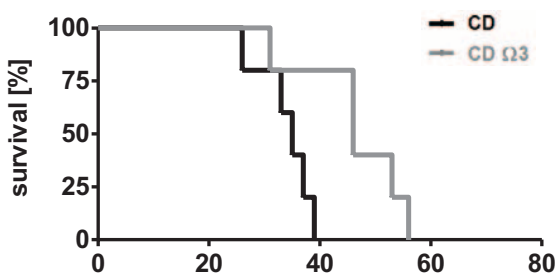

D
MC-38 cell injection after treatment

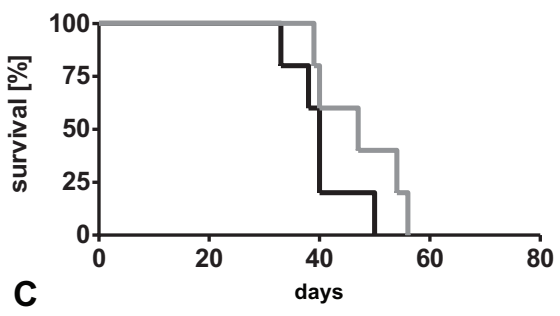

MC-38 cell injection after treatment

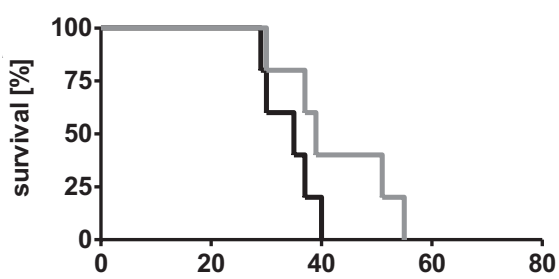

$\mathbf{E}$ after treatment.

HFD- $\Omega 3 F$ F animals, indicating $\Omega 3 F$ F delay CRLM development as confirmed on MRI (Supplementary Figure 3, http://links.lww.com/ SLA/B84). Therefore, $\Omega 3 F A$ maintain some anticancer effects in liver also after treatment cessation.

Above experiments were repeated in mice on a CD. For inoculation before treatment, survival was 35 days in $\mathrm{CD}$ and 46 days in CD- $\Omega 3$ FA mice $[P=0.027$; liver weights $3.6 \mathrm{~g}( \pm 0.9)$ and $4.5 \mathrm{~g}( \pm 1.5)$, respectively]. For inoculation after treatment, survival was 35 days in $\mathrm{CD}$ and 39 days in $\mathrm{CD}-\Omega 3 \mathrm{FA}$ mice $[P=0.137$; liver weights $4.8 \mathrm{~g}( \pm 1.9)$ and $4.3 \mathrm{~g}( \pm 1.3)$, respectively]. Therefore, $\Omega 3 F A$ may also inhibit CRLM in lean liver, albeit modestly.

We conclude that $\Omega 3 F$ A may exert anticancer effects on both fatty and lean liver even beyond their administration. $\Omega 3 F A$ are hence unlikely to compromise oncological outcomes in liver surgery.

\section{DISCUSSION}

In this study, we showed in a mouse model of HFD-induced NAFLD that preoperative $\Omega 3$ FA treatment confers several benefits influencing the outcome of major surgery. We observed that steatotic reduction associated with the use of $\Omega 3 \mathrm{FA}$ has relevant positive consequences on (i) the sensitivity of liver to I/R, (ii) its regenerative capacity following tissue loss, and (iii) seemingly also on CRLM, a prime indication for liver surgery. Furthermore, we found that $\Omega 3 \mathrm{FA}$ maintain - at a smaller scale - these beneficial effects in the absence of steatosis, indicating the mode of action of $\Omega 3 \mathrm{FA}$ extends beyond the sole reduction of fat. Therefore, $\Omega 3 F A$ may suit to expand the application of liver surgery in situations of both fatty and nonfatty liver surgery.

The health benefits of $\Omega 3 \mathrm{FA}$ are manifold and cannot be attributed to a single mechanism. Dietary $\Omega 3 F A$ comprise a number of fatty acids, of which EPA and DHA are the major constituents. Apart from modulating the composition and function of cell membranes, $\Omega 3 F A$ can activate specific receptors (eg, GPR $120^{12}$ ) and are further metabolized into a series of bioactive lipids (eg, resolvins, protectins), which participate in cellular signaling and are intermingled with other lipid signaling systems, such as by acting as ligands of cannabinoidtype receptors. ${ }^{27,28}$ This underlying complexity suggests that detailed action mechanisms of $\Omega 3 F$ F will vary to provide dynamic responses toward specific situations. More broadly, $\Omega 3 F A$ however do have phenotypic effects that may be considered as general attributes of these lipids. The anti-inflammatory action (eg, nuclear factor kappa-lightchain-enhancer of activated B cells (NFKB) inhibition), the promotion of a healthy endothelial phenotype (eg, NO production), and the changes in energy turnover (eg, promotion of $\beta$-oxidation, inhibition of mammalian target of rapamycin [mTOR] signaling) reminiscent of a fasting response (eg, activation of silent mating type information regulation 2 homolog 1 [SIRT1], 5' adenosine monophosphate-activated protein kinase [AMPK]) likely are properties of $\Omega 3 \mathrm{FA}$ that contribute to their beneficial effects on steatosis, hepatic I/R, liver regeneration, and cancer. ${ }^{9,29-32}$

Inflammation, endothelial dysfunction, and alterations in oxidative metabolism have all been causally linked to steatosis. ${ }^{10,33,34}$ We have previously shown that relieving steatosis-associated vasoconstriction via $\Omega 3$ FA normalizes the oversensitivity toward $I / R,{ }^{13}$ while $\Omega 3 F A-G P R 120$ mediated inhibition of inflammatory Kupffer cells protects lean liver akin to fasting. ${ }^{12,25}$ Endothelial effects clearly will add, because sinusoids are the first to experience the consequences of ischemia. Proper endothelial function likewise is a prerequisite for liver regeneration after resection, ${ }^{35}$ and the improvement of the defective regeneration seen in aged liver is associated with the reinstallation of vascular integrity. ${ }^{36}$ How $\Omega 3$ FA's anti-inflammatory 
action affects liver regeneration is unclear; for example, secretion of tumor necrosis factor (TNF) from Kupffer cells is needed for regeneration, ${ }^{37}$ but excess TNF, often elevated in fatty liver, ${ }^{38}$ causes liver failure after hepatectomy. ${ }^{39}$ Furthermore, targeting inflammatory Kupffer cells has a little effect on regeneration, ${ }^{40}$ suggesting that inflammatory contributions to regeneration are more complex than thought.

Inflammation and pathological angiogenesis are hallmarks of cancer; the trend seen for $\Omega 3 F$ F in inhibiting CRLM is hence not surprising. ${ }^{32,41}$ A tendency to prolonged survival through $\Omega 3 \mathrm{FA}$ was observed in all 4 settings (HFD vs CD, injection before vs after treatment) we tested, indicating that $\Omega 3 \mathrm{FA}$ may be beneficial, but certainly not harmful, with regard to CRLM. The largest effect on survival was seen when cancer cells were injected before treatment, thus when the early CRLM development was fully exposed to $\Omega 3 \mathrm{FA}$. When injected after treatment, $\Omega 3 F A$ levels are expected to decline over time, explaining their diminished "anticancer" effects. Of note, survival in general was shortened in lean versus fatty liver, perhaps suggesting an anti-malignant effect for steatosis. We speculate this was due to better perfusion in lean liver, ${ }^{13}$ facilitating migration/ transport of cancer cells from the portal vein into the sinusoids and thereby increasing the initial malignant load. Although controversial, some clinical observations have reported a reduced incidence of CRLM in steatotic patients and an improved survival in steatotic/ obese CRLM patients, ${ }^{42-45}$ consistent with steatosis creating a microenvironment unfavorable to invasion and colonization of liver by malignant intestinal cells.

Altogether, $\Omega 3 \mathrm{FA}$ treatment of fatty and lean liver was beneficial across the key aspects related to major liver surgery. To foster a potential clinical application, we have initiated a clinical trial (NCT01884948) to assess the impact of $\Omega 3 \mathrm{FA}$ on outcomes after major liver surgery. This study should also inform on the $\Omega 3 F A$ activities in I/R, regeneration, and steatosis in patients. ${ }^{46}$

The use of $\Omega 3 \mathrm{FA}$ as a measure to expand the application of surgery to fatty liver, or lean liver requiring extended resection, comes with several advantages; the pre-operative administration of $\Omega 3 F A$ is simple, either as a food supplement or via intravenous injection (Omegaven). Therefore, little issues with compliance are expected, unlike for the currently recommended NAFLD treatment, that is, exercise combined with caloric restriction. ${ }^{31}$ Given that $\Omega 3 F A$ are being ingested as part of our food and have been assessed in various trials, potential side effects will not hamper their clinical use. ${ }^{47}$ Indeed, prolonged application such as needed for "defattening" may exert additional benefits, including the mitigation of the MS, cardiovascular disease, or other comorbidities associated with NAFLD. ${ }^{31}$ More research is needed to identify which of the $\Omega 3 F A$ lipids and at what dosages may induce specific benefits in a given setting.

In conclusion, our findings suggest a potential of $\Omega 3 F A$ in extending surgical options for both fatty and lean livers. Apart from the steatotic changes, the benefits of $\Omega 3 F$ A include an increased tolerance toward ischemic reperfusion injury, an improved regenerative capacity, and a trend toward lower mortality due to liver tumors. These effects partially are not only a consequence of the $\Omega 3 F A$ associated defattening but also occur in the absence of significant steatosis, implying that the anti-inflammatory, vascular, and metabolic effects of these lipids are likewise beneficial to physiological liver function. Their ease of application, their safety profile, their affordability, and their many potential health benefits with regard to comorbidities or oncological indications advocate their use in the clinic. Considering the endemic rise in fatty liver disease, $\Omega 3 F A$ based strategies hold a strong position in improving outcomes and should be tested in convincing clinical studies.

\section{ACKNOWLEDGMENT}

We would like to thank Udo Ungethüm, Eleonora Maurizio, and Pia Fuchs for the excellent technical assistance.

\section{REFERENCES}

1. Malik VS, Willett WC, Hu FB. Global obesity: trends, risk factors and policy implications. Nat Rev Endocrinol. 2013;9:13-27.

2. Humar B, Semmo N. Endocannabinoid signaling in liver pathologies. In: Dufour JF, Clavien PA, eds. Signaling Pathways in Liver Disease. Oxford: Wiley Blackwell; 2015:226-239.

3. Clavien PA, Petrowsky H, DeOliveira ML, et al. Strategies for safer liver surgery and partial liver transplantation. N Engl J Med. 2007;356:1545-1559.

4. Selzner M, Clavien PA. Failure of regeneration of the steatotic rat liver: disruption at two different levels in the regeneration pathway. Hepatology. 2000;31:35-42.

5. McCormack L, Petrowsky H, Jochum W, et al. Hepatic steatosis is a risk factor for postoperative complications after major hepatectomy: a matched casecontrol study. Ann Surg. 2007;245:923-930.

6. Risk and Prevention Study Collaborative Group. n-3 fatty acids in patients with multiple cardiovascular risk factors. N Engl J Med. 2013;368:1800-1808.

7. Kim HJ, Takahashi M, Ezaki O. Fish oil feeding decreases mature sterol regulatory element-binding protein 1 (SREBP-1) by down-regulation of SREBP-1c mRNA in mouse liver. A possible mechanism for down-regulation of lipogenic enzyme mRNAs. J Biol Chem. 1999;274:25892-25898.

8. Ruiz-Gutierrez V, Perez-Espinosa A, Vazquez CM, et al. Effects of dietary fats (fish, olive and high-oleic-acid sunflower oils) on lipid composition and antioxidant enzymes in rat liver. Br J Nutr. 1999;82:233-241.

9. Zuniga J, Cancino M, Medina F, et al. N-3 PUFA supplementation triggers PPAR-alpha activation and PPAR-alpha/NF-kappaB interaction: antiinflammatory implications in liver ischemia-reperfusion injury. PLoS One 2011;6:e28502.

10. Fedor DM, Adkins Y, Mackey BE, et al. Docosahexaenoic acid prevents trans10, cis-12-conjugated linoleic acid-induced nonalcoholic fatty liver disease in mice by altering expression of hepatic genes regulating fatty acid synthesis and oxidation. Metab Syndr Relat Disord. 2012;10:175-180.

11. Capanni M, Calella F, Biagini MR, et al. Prolonged n-3 polyunsaturated fatty acid supplementation ameliorates hepatic steatosis in patients with nonalcoholic fatty liver disease: a pilot study. Aliment Pharmacol Ther 2006;23:1143-1151.

12. Raptis DA, Limani P, Jang JH, et al. GPR120 on Kupffer cells mediates hepatoprotective effects of omega3-fatty acids. J Hepatol. 2014; 60:625-632.

13. El-Badry AM, Jang JH, Elsherbiny A, et al. Chemical composition of hepatic lipids mediates reperfusion injury of the macrosteatotic mouse liver through thromboxane A(2). J Hepatol. 2011;55:1291-1299.

14. Qiu YD, Wang S, Yang Y, et al. Omega-3 polyunsaturated fatty acids promote liver regeneration after $90 \%$ hepatectomy in rats. World J Gastroenterol. 2012;18:3288-3295.

15. Marsman HA, de Graaf W, Heger M, et al. Hepatic regeneration and functional recovery following partial liver resection in an experimental model of hepatic steatosis treated with omega-3 fatty acids. Br J Surg. 2013; 100:674-683.

16. Ibrahim ES, Saleh SM, El Hoseeny M, et al. Effect of omega-3 on hepatic regeneration in adult living donors undergoing hepatic resections for liver transplantation: a randomized controlled trial. J Crit Care. 2016; 31:157-162.

17. D'Eliseo D, Velotti F. Omega-3 fatty acids and cancer cell cytotoxicity: implications for multi-targeted cancer therapy. J Clin Med. 2016;5;pii: E15.

18. Griffini P, Fehres O, Klieverik L, et al. Dietary omega-3 polyunsaturated fatty acids promote colon carcinoma metastasis in rat liver. Cancer Res. 1998;58:3312-3319.

19. Gutt CN, Brinkmann L, Mehrabi A, et al. Dietary omega-3-polyunsaturated fatty acids prevent the development of metastases of colon carcinoma in rat liver. Eur J Nutr. 2007;46:279-2785.

20. Raptis DA, Fischer MA, Graf R, et al. MRI: the new reference standard in quantifying hepatic steatosis? Gut. 2012;61:117-1127.

21. Van Handel E. Rapid determination of total lipids in mosquitoes. J Am Mosq Control Assoc. 1985;1:302-304.

22. Oberkofler CE, Limani P, Jang JH, et al. Systemic protection through remote ischemic preconditioning is spread by platelet-dependent signaling in mice. Hepatology. 2014;60:1409-1417. 
23. Lehmann K, Tschuor C, Rickenbacher A, et al. Liver failure after extended hepatectomy in mice is mediated by a p21-dependent barrier to liver regeneration. Gastroenterology. 2012;143:1609-1619e4.

24. Limani P, Borgeaud N, Linecker M, et al. Selective portal vein injection for the design of syngeneic models of liver malignancy. Am J Physiol Gastrointest Liver Physiol 2016; 310:G682-G688.

25. Rickenbacher A, Jang JH, Limani P, et al. Fasting protects liver from ischemic injury through Sirt1-mediated downregulation of circulating HMGB1 in mice. J Hepatol. 2014;61:301-308.

26. Marsman HA, Heger M, Kloek JJ, et al. Omega-3 fatty acids reduce hepatic steatosis and consequently attenuate ischemia-reperfusion injury following partial hepatectomy in rats. Dig Liver Dis. 2011;43:984-990.

27. Brown I, Cascio MG, Rotondo D, et al. Cannabinoids and omega-3/6 endocannabinoids as cell death and anticancer modulators. Prog Lipid Res. 2013;52:80-109.

28. Wang W, Zhu J, Lyu F, et al. omega-3 polyunsaturated fatty acids-derived lipid metabolites on angiogenesis, inflammation and cancer. Prostaglandins Other Lipid Mediat. 2014;113-115:13-20.

29. Xue B, Yang Z, Wang X, et al. Omega-3 polyunsaturated fatty acids antagonize macrophage inflammation via activation of AMPK/SIRT1 pathway. PLoS One. 2012;7:e45990.

30. Wang Q, Liang X, Wang L, et al. Effect of omega-3 fatty acids supplementation on endothelial function: a meta-analysis of randomized controlled trials. Atherosclerosis. 2012;221:536-543.

31. Di Minno MN, Russolillo A, Lupoli R, et al. Omega-3 fatty acids for the treatment of non-alcoholic fatty liver disease. World $J$ Gastroenterol. 2012;18:5839-5847.

32. Manzi L, Costantini L, Molinari R, et al. Effect of dietary omega-3 polyunsaturated fatty acid DHA on glycolytic enzymes and Warburg phenotypes in cancer. Biomed Res Int. 2015;2015:137097.

33. Louvet A, Teixeira-Clerc F, Chobert MN, et al. Cannabinoid CB2 receptors protect against alcoholic liver disease by regulating Kupffer cell polarization in mice. Hepatology. 2011;54:1217-1226.

34. Maslak E, Gregorius A, Chlopicki S. Liver sinusoidal endothelial cells (LSECs) function and NAFLD; NO-based therapy targeted to the liver. Pharmacol Rep. 2015;67:689-694.
35. Ding L, Saunders TL, Enikolopov G, et al. Endothelial and perivascular cells maintain haematopoietic stem cells. Nature. 2012;481:457-462.

36. Furrer K, Rickenbacher A, Tian Y, et al. Serotonin reverts age-related capillarization and failure of regeneration in the liver through a VEGFdependent pathway. Proc Natl Acad Sci U S A. 2011;108:2945-2950.

37. Varela-Rey M, Beraza N, Lu SC, et al. Role of AMP-activated protein kinase in the control of hepatocyte priming and proliferation during liver regeneration. Exp Biol Med (Maywood). 2011;236:402-408.

38. Seo YY, Cho YK, Bae JC, et al. Tumor necrosis factor-alpha as a predictor for the development of nonalcoholic fatty liver disease: a 4-year follow-up study. Endocrinol Metab (Seoul). 2013;28:41-45.

39. Mohammed FF, Smookler DS, Taylor SE, et al. Abnormal TNF activity in Timp3-/- mice leads to chronic hepatic inflammation and failure of liver regeneration. Nat Genet. 2004;36:969-977.

40. Jepsen BN, Andersen KJ, Knudsen AR, et al. Anti-inflammatory liposomes have no impact on liver regeneration in rats. Ann Med Surg (Lond). 2015;4:452-461.

41. Nabavi SF, Bilotto S, Russo GL, et al. Omega-3 polyunsaturated fatty acids and cancer: lessons learned from clinical trials. Cancer Metastasis Rev. 2015;34:359-380.

42. Hayashi S, Masuda H, Shigematsu M. Liver metastasis rare in colorectal cancer patients with fatty liver. Hepatogastroenterology. 1997;44:1069-1075.

43. Murono K, Kitayama J, Tsuno NH, et al. Hepatic steatosis is associated with lower incidence of liver metastasis from colorectal cancer. Int J Colorectal Dis. 2013;28:1065-1072.

44. Parkin E, O'Reilly DA, Adam R, et al. The effect of hepatic steatosis on survival following resection of colorectal liver metastases in patients without preoperative chemotherapy. HPB (Oxford). 2013;15:463-472.

45. Amptoulach S, Gross G, Kalaitzakis E. Differential impact of obesity and diabetes mellitus on survival after liver resection for colorectal cancer metastases. J Surg Res. 2015;199:378-385.

46. Linecker M, Limani P, Botea F, et al. "A randomized, double-blind study of the effects of omega-3 fatty acids (Omegaven) on outcome after major liver resection". BMC Gastroenterol. 2015;15:102.

47. Lenox CE, Bauer JE. Potential adverse effects of omega-3 fatty acids in dogs and cats. J Vet Intern Med. 2013;27:217-226. 